\title{
$\beta$-Alanine supplemented diets enhance behavioral resilience to stress exposure in an animal model of PTSD
}

\author{
Jay R. Hoffman • Ishay Ostfeld • Jeffrey R. Stout • \\ Roger C. Harris · Zeev Kaplan · Hagit Cohen
}

Received: 23 February 2015 / Accepted: 25 February 2015 / Published online: 11 March 2015

(C) The Author(s) 2015. This article is published with open access at Springerlink.com

\begin{abstract}
This study investigated the effects of $\beta$-alanine (BA) ingestion on the behavioral and neuroendocrine response of post-traumatic stress disorder (PTSD) in a murine model. Animals were fed a normal diet with or without (PL) BA supplementation (100 $\left.\mathrm{mg} \mathrm{kg}^{-1}\right)$ for 30 days. Animals were then exposed to a predator-scent stress (PSS) or a sham (UNEX). Behaviors were evaluated using an elevated plus maze (EPM) and acoustic startle response (ASR) 7 days following exposure to the PSS. Corticosterone concentrations (CS), expression of brain-derived neurotrophic factor (BDNF), and brain carnosine concentrations were analyzed a day later. Animals in PSS+PL spent significantly less time in the open arms and in the number of entries in the EPM than PSS+BA, UNEX+BA, or UNEX+PL. Animals in PSS+BA had comparable scores to UNEX+BA. Anxiety index was higher $(p<0.05)$ in PSS+PL compared to PSS+BA or animals that were unexposed. ASR and freezing were greater $(p<0.05)$ in animals exposed to PSS compared to animals unexposed. CS expression was
\end{abstract}

Handling Editor: G. Lubec.

J. R. Hoffman $(\bowtie) \cdot J$. R. Stout

Institute of Exercise Physiology and Wellness, Sport and Exercise

Science, University of Central Florida, Orlando, FL 32816, USA

e-mail: jay.hoffman@ucf.edu

I. Ostfeld

Israel Defense Force, Medical Corps, Tel Hashomer, Israel

R. C. Harris

Junipa Ltd, Newmarket, UK

Z. Kaplan $\cdot$ H. Cohen

Anxiety and Stress Research Unit, Beer-Sheva Mental Health Center, Faculty of Health Sciences, Division of Psychiatry, Ben-

Gurion University of the Negev, Beer-Sheva, Israel higher $(p<0.05)$ in animals exposed to PSS compared to unexposed animals. Brain carnosine concentrations in the hippocampus and other brain sections were significantly greater in animals supplemented with BA compared to PL. BDNF expression in the CA1 and DG subregions of the hippocampus was lower $(p<0.05)$ in animals exposed and fed a normal diet compared to animals exposed and supplemented with BA, or animals unexposed. In conclusion, BA supplementation in rats increased brain carnosine concentrations and resulted in a reduction in PTSD-like behavior, which may be mediated in part by maintaining BDNF expression in the hippocampus.

Keywords Supplementation - Carnosine $\cdot$ Military Nutrition $\cdot$ BDNF $\cdot$ Corticosterone

\section{Introduction}

Stress resulting from an acute traumatic experience can result in a variety of manifestations that include recurring and unwanted recollections or dreams of the event that cause significant behavioral changes (American Psychiatric Association 2013). Responses from acute stress may include avoidance of feelings or reminders of the event, marked arousal including irritability, hypervigilance, an elevated startle response, a concentration deficit or emotional numbing (American Psychiatric Association 2013). Although these responses to acute stress are often used to diagnose post-traumatic stress disorder (PTSD) (American Psychiatric Association 1994), several studies have indicated that some individuals are able to adapt within a short time period following the traumatic experience and not experience PTSD (Bisson et al. 2004; Bryant et al. 2008). Bryant et al. (2014) demonstrated that identification of an 
acute stress disorder within the first month of the traumatic experience appears to be only moderately sensitive (51 and $45 \%$ within 3 and 12 months, respectively) for predicting PTSD. However, the absence of acute stress disorder appears to be highly predictive (95 and $94 \%$ within 3 and 12 months, respectively) that the individual will not develop PTSD (Bryant et al. 2014).

The pathophysiology of PTSD is thought to be related to changes in the structure of neurons within areas of the brain that control stress and memory (McEwen 2007). The hippocampus is one of the most sensitive and adaptable regions of the brain, and many of the changes within the hippocampus occur within the dentate gyrus (DG)-CA3 region (McEwen 2007). The DG-CA3 region is thought to play a role in the memory of sequences of events, and several changes have been suggested to occur in this region during stress such as a suppression of neurogenesis or cell survival, and a degeneration of dendrites and synapses (McEwen 1999; Sousa et al. 1998; Stewart et al. 2005). The effect of stress on brain function though is not limited to the hippocampus only. The hippocampus works synergistically with the cortex and amygdala to control the processing of emotional memories (Richardson et al. 2004) and reduction of fear (Milad and Quirk 2002). Although the mechanism that is stimulating the structural remodeling of neurons in the brain is quite complex, evidence is compelling that changes in circulating glucocorticoids (McEwen 2007; Myers et al. 2014) and expression of brain-derived neurotrophic factor (BDNF) (Yao et al. 2011) are closely linked to the plasticity of brain function.

Stress has a profound influence on changes in the neuroendocrine system resulting in a significant elevation in glucocorticoids (Myers et al. 2014). Glucocorticoids are released from the adrenal gland and cross the blood-brain barrier to bind to receptors in neurons or glia cells. Glucocorticoid receptors are found in high concentrations within the brain, specifically in areas that are related to sites of stress such as the hippocampus, amygdala, and frontal cortex (Fuxe et al. 1987; Myers et al. 2014). Corticosterone injections in the dorsal hippocampus of rodents have been demonstrated to cause PTSD-like memory impairments that accompany impaired hippocampal function (Kaouane et al. 2012). Elevations in glucocorticoids are generally associated with dendritic remodeling and memory that often resemble what is seen during chronic stress (Miller and McEwen 2006). However, the glucocorticoid response to stress appears to follow an inverted U pattern. Very low or very high concentrations of circulating glucocorticoids during periods of stress are more likely to negatively alter neural plasticity (Miller and McEwen 2006).

BDNF is part of the neurotrophin family and has been demonstrated to have an important role in neuronal remodeling and modulating synaptic plasticity and neurotransmitter release (Castrén and Rantamäki 2010). Angelucci et al. (2014) recently compared BDNF concentrations in individuals diagnosed with PTSD to individuals who were exposed to a traumatic event but not diagnosed with PTSD. Serum BDNF concentrations were significantly lower in PTSD patients compared to the control subjects. In a rodent model, exposure to stress has been shown to down-regulate BDNF mRNA expression (Kozlovsky et al. 2007). Increasing BDNF expression, or decreasing glucocorticoid levels in individuals who are experiencing stress, may provide increased resiliency to PTSD following exposure to trauma.

Treatment for PTSD is quite varied and generally involves a combination of psychotherapy and pharmacological options that occur following diagnosis (Kirkpatrick and Heller 2014). However, less information is available regarding potential options for preventing or increasing resiliency to PTSD. Murakami and Furuse (2010) reported that a $\beta$-alanine supplemented diet in mice was able to increase brain carnosine concentrations in the cerebral cortex and hypothalamus and increase the concentration of BDNF in the hippocampus. These changes were also accompanied by significantly greater activity of the mice in the open arms of an elevated plus-maze test. Although $\beta$-alanine is considered to act as an inhibitory neurotransmitter that can cross the blood-brain barrier (Takeuchi et al. 2000), the lack of any significant change in $\beta$-alanine concentrations in the cerebral cortex and hypothalamus suggests that the anxiolytic effects observed are likely related to an elevation in brain carnosine ( $\beta$-alanyl-L-histidine) concentrations. This is supported by others demonstrating that elevated brain carnosine can induce antidepressant-like activity (Tomonaga et al. 2004, 2008).

In consideration that $\beta$-alanine can increase brain carnosine concentrations, which may subsequently induce antidepressant activity, the primary purpose of this study was to examine the effect of 30 days of $\beta$-alanine ingestion on PTSD-like behavioral changes in rodents exposed to a predator-scent stress (PSS). A secondary purpose was to investigate the mechanisms underlying the potential beneficial effects of $\beta$-alanine ingestion by examining circulating corticosterone concentrations and BDNF expression in the hippocampus.

\section{Methods}

Animals

Adult male Sprague-Dawley rats weighing 200-250 g ( $n=122)$ were habituated to housing conditions for at least 7 days. All animals were housed four per cage in a vivarium with stable temperature and a reversed 12-h light/dark 
cycle, with unlimited access to food and water. $\beta$-Alanine was provided with glucomannan in a powder form in an 80:20 blend. Rats were provided with $100 \mathrm{mg}$ of the powder per $\mathrm{kg}$ of body mass (a total of $30 \mathrm{mg}$ of powder was dissolved in $25 \mathrm{~mL}$ of water). PL-treated rats were provided with the vehicle (glucommanan) at the same relative dose. Animals were handled once daily. All testing was performed during the dark phase in dim red light conditions. This study was performed according to the principles and guidelines of the National Institute of Health Guide for the Care and Use of Laboratory Animals. All treatment and testing procedures were approved by the Animal Care Committee of the Ben-Gurion University of the Negev, Israel.

\section{Experimental design}

Rats were randomly assigned to one of four treatment groups ( $n=30$ or 31 per group):

1. Unexposed and vehicle-treated group (UNEXP+PL): rats were fed regular food and water for 30 days and were exposed to fresh, unused litter for $15 \mathrm{~min}$.

2. Unexposed and treated with $\beta$-alanine (UNEXP+BA): rats were provided $\beta$-alanine in their water and were exposed to fresh, unused litter for $15 \mathrm{~min}$.

3. Exposed and vehicle-treated (EXP+PL): rats were fed regular food and water for 30 days and were exposed to PSS for $15 \mathrm{~min}$.

4. Exposed and treated with $\beta$-alanine (EXP+BA): rats were provided $\beta$-alanine in their water exposed to PSS.

Following the 7-day acclimation period in which all rats received a normal powder diet, they were randomized into four groups. Following 30 days of either normal diet or $\beta$-alanine supplemented diet the rats were exposed to either the PSS or sham protocol. All behavioral tests were conducted 7 days following the PSS or sham protocol, and the rats were then sacrificed $24 \mathrm{~h}$ later and brains were removed. Diets were maintained until the end of the study. The validity of this model has been demonstrated in several studies (Cohen et al. 2004, 2012b; Kozlovsky et al. 2007).

Predator-scent stress (PSS)

Following the 30-day supplementation regimen, animals were exposed to the PSS protocol. The PSS protocol consisted of placing the experimental animal on well-soiled cat litter (in use by the cat for 2 days, sifted for stools) for $10 \mathrm{~min}$ in a closed environment. Control animals were exposed to fresh, unused litter for the same amount of time. The situational reminder consisted of placing animals on fresh, unused cat litter for $15 \mathrm{~min}$.

\section{Assessment schedule}

Behavioral responses were assessed in the elevated plusmaze, acoustic startle response, and contextual freezing. All results were recorded and analyzed using an EthoVision automated tracking system (Noldus Information Technology, The Netherlands). Performance in the elevated plusmaze and acoustic startle response occurred 7-days following the initial exposure to the PSS. The contextual freezing measures were performed on day 8 following initial exposure. The delay in performing these measures from the PSS is based upon findings that extreme behavioral changes, which remain constant after 7 days of exposure and represent 'chronic symptoms' (Cohen et al. 2004) which persist over a prolonged duration (Cohen and Zohar 2004; Cohen et al. 2004). Following behavioral assessments, all animals were killed and their brains removed for analysis.

\section{Behavioral measures}

\section{Elevated plus-maze (EPM)}

Behavioral assessments performed in the EPM have previously been described (Cohen et al. 2003, 2012a, b). The EPM is a plus-shaped platform with two opposing open and two opposing closed arms (open only towards the central platform and surrounded by $14-\mathrm{cm}$ high opaque walls on three sides). Rats were placed on the central platform facing an open arm and allowed to explore the maze for $5 \mathrm{~min}$. Each session was videotaped and subsequently scored by an independent observer. Arm entry was defined as entering an arm with all four paws. The following behaviors were assessed: time spent (duration) in open and closed arms and on the central platform; number of open and closed arm entries; and total exploration (entries into all arms). Total exploration was calculated as the number of entries into any arm of the maze to distinguish between impaired exploratory behavior, exploration limited to closed arms (avoidance), and free exploration. "Anxiety Index", an index that integrates the EPM behavioral measures, was calculated as follows:

Anxiety Index $=1-\left[\frac{\left(\frac{\text { time spent in the open arms }}{\text { total time on the maze }}\right)+\left(\frac{\text { number of entries to the open arms }}{\text { total exploration on the maze }}\right)}{2}\right]$. 
Anxiety Index values range from 0 to 1 where an increase in the index expresses increased anxiety-like behavior.

\section{Acoustic startle response}

Startle response was measured using two ventilated startle chambers (SR-LAB system, San Diego Instruments, San Diego, CA). The SR-LAB calibration unit was used routinely to ensure consistent stabilimeter sensitivity between test chambers and over time. Each Plexiglas cylinder rests on a platform inside a sound-proofed, ventilated chamber. Movement inside the tube is detected by a piezoelectric accelerometer below the frame. Sound levels within each test chamber are measured routinely using a sound level meter to ensure consistent presentation. Each test session started with a 5-min acclimatization period to background white noise of $68 \mathrm{~dB}$, followed by 30 acoustic startle trial stimuli in six blocks ( $110 \mathrm{~dB}$ white noise of $40 \mathrm{~ms}$ duration with 30 or $45 \mathrm{~s}$ inter-trial interval). Behavioral assessment consisted of mean startle amplitude (averaged over all 30 trials) and percent of startle habituation to repeated presentation of the acoustic pulse. Percent habituation-the percent change between the response to the first block of sound stimuli and the last—was calculated as follows:
Measurement of serum corticosterone

Serum corticosterone was measured with ELISA Test Kit (Endocrine Technologies Inc. Newark, CA) according to the instructions of the manufacturer. Plates were read at $450 \mathrm{~nm}$. All reactions were determined in duplicate. Interassay variation was $6.1 \%$ and intra-assay variation $5.9 \%$. All samples were measured in duplicate. The lowest level detectable in this assay is $0.1 \mathrm{ng} / \mathrm{mL}$ of serum.

\section{Brain BDNF levels}

\section{Tissue preparation}

Twenty-four hours following the behavioral tests, ten animals from each group were deeply anesthetized (ketamine and xylazine mixture) and perfused transcardially with cold $0.9 \%$ physiological saline followed by $4 \%$ paraformaldehyde (Sigma-Aldrich) in $0.1 \mathrm{M}$ phosphate buffer ( $\mathrm{pH}$ 7.4). Brains were quickly removed, postfixed in the same fixative for $12 \mathrm{~h}$ at $4{ }^{\circ} \mathrm{C}$, and cryoprotected overnight in $30 \%$ sucrose in $0.1 \mathrm{M}$ phosphate buffer at $4{ }^{\circ} \mathrm{C}$. Brains were frozen on dry ice and stored at $-80{ }^{\circ} \mathrm{C}$. Serial coronal sections $(10 \mu \mathrm{m})$ at the level

Percent Habituation $=100 \times\left[\frac{(\text { average startle amplitude in Block } 1)-(\text { average startle amplitude in Block 6) }}{\text { average startle amplitude in Block } 1}\right]$.

Contextual freezing measurement

Freezing behavior was scored during the situational reminder/cue exposure and was defined as an absence of all movement (except for respiration) (Kim et al. 1992). Total cumulative freezing time (total seconds spent freezing during each assessment period) was measured and calculated as a percentage of total time. Freezing behavior was recorded using an overhead video camera and scored for immobility using the recorded images. The videotape and the recorded images were both scored by a trained observer unaware of the treatment conditions.

\section{Blood sampling}

Twenty rats from each group were decapitated with a guillotine. Care was taken to minimize situational stress: the area was thoroughly cleaned between each killing and bodies were removed. Trunk blood was collected and left at room temperature for $2 \mathrm{~h}$ and then centrifuged (1000g for 10 min at $4{ }^{\circ} \mathrm{C}$ ) with a Hermle centrifuge. Serum (approximately $1 \mathrm{~mL}$ from each rat) was collected and stored at $-80{ }^{\circ} \mathrm{C}$ until the analysis was performed. of dorsal hippocampus were collected for each animal, using a cryostat (Leica CM 1850) and mounted on coated slides.

\section{Immunofluorescence}

Sliced sections were air dried and incubated in frozen methanol ( $2 \mathrm{~min})$ and in $4 \%$ Para-formal-aldehyde (4 min). After three washes in phosphate-buffered saline (PBS) containing Tween 20 (PBS/T) (Sigma-Aldrich), the sections were incubated for $60 \mathrm{~min}$ in a blocking solution in normal goat or horse serum in PBS and then overnight at $4{ }^{\circ} \mathrm{C}$ with the primary antibodies against BDNF $(1: 250$ each; Abcam). After three washes in PBS/T, sections were incubated in DyLight-488 in PBS containing $2 \%$ normal serum for $2 \mathrm{~h}$. Sections were washed and mounted with mounting medium (Vectrastain Vector laboratories, USA). Control staining was performed in the absence of the primary antibodies. Additionally, secondary fluorescent labels were swapped to check cross-reactivity and sections were incubated without any primary antibodies to check for any non-specific binding of the secondary antibodies. 


\section{Quantification}

A computer-assisted image analysis system (Leica Application Suite V3.6, Leica, Germany) was used for quantitative analysis of the immunostaining and $50 \times$ objective lens was employed to assess the number of BDNF-IR positive cells in the hippocampus, divided into three (counted separately) areas: CA1 subfield, CA3 subfield, and dentate gyrus (DG). The regions of interest were outlined and computer-aided estimation was used to calculate the number of BDNF-IR cells in the pyramidal layer of CA1 and CA3, and in the granular layer of the DG. Seven representative sections of the hippocampus were chosen (between Bregma -2.30 and Bregma -3.60) from each animal, from each group. The sections were analyzed by two observers blinded to the treatment protocol. Standard technique was used to estimate the number of BDNF cell profiles per unit area for each investigated hippocampal structure.

\section{Measurement of brain carnosine concentrations}

Carnosine concentrations in brain homogenates were determined by Liquid Chromatographic/tandem mass spectrometric (LC/MS/MS) analysis according to previously published methods (Aldini et al. 2004). Brains were partially thawed on ice and six brain regions were sampled: cerebral cortex, hypothalamus, hippocampus, amygdala, olfactory bulb, and thalamus. Each sample was weighed and transferred into individual vials for further homogenization.

Brain samples were homogenized in saline $(0.9 \%$ $\mathrm{NaCl} ; 100 \mathrm{mg} \mathrm{mL}^{-1}$ ) supplemented with $1 \mathrm{mM}$ EDTA, using Polytron Homogenizer. Homogenates were stored at $-20 \pm 5{ }^{\circ} \mathrm{C}$ prior to LC/MS/MS analysis. Homogenates were spiked with Internal Standard (D3-L-Dopa) and de-proteinized by addition of $100 \mu \mathrm{L}$ of $700 \mathrm{mM}$ PCA to $250 \mu \mathrm{L}$ sample. After $1 \mathrm{~min}$ of Vortex, samples were centrifuged at 13,200 RPM [15.7 Relative Centrifugal Force (RCF)] for $12 \mathrm{~min}$. The supernatant was diluted with $100 \mu \mathrm{L}$ of Mobile phase and injected. L-Carnosine purchased from Sigma was used to prepare standards for the calibration curve (in ppb). Samples were diluted $\times 5$ prior to analysis. The concentration read from the standard curve was multiplied by the dilution factor and converted to ppm units.

\section{Statistical analyses}

The statistical analyses were performed using a one-way analysis of variance (ANOVA). In the event of a significant $\mathrm{F}$ ratio, LSD post hoc analysis was used for pairwise comparisons. All data are reported as mean \pm SD. Pearson's product-moment correlation was used to determine selected bivariate correlations. An alpha level of $p<0.05$ was used to determine statistical significance. Data were analyzed using SPSS v22 software (SPSS Inc., Chicago, IL).

\section{Results}

Elevated plus-maze

Comparisons between the groups for time spent in open arms indicated a significant effect $(F(3,118)=7.06$, $p<0.001)$. Post-hoc analyses indicated that animals that consumed a normal diet and were exposed to PSS spent significantly less time in the open arms than all other treatment groups ( $p$ value ranging from $<0.001$ to 0.012 ). No other between-group differences were noted in time spent in open arms (see Fig. 1a). Comparisons of open arm entries (see Fig. 1b) also showed a significant interaction between groups $(F(3,118)=9.55, p<0.001)$. Normal diet fed animals that were exposed to PSS exhibited significantly ( $p$ value $<0.001$ ) fewer entries than all other treatment groups. ( $p$ value $<0.001$ ). No other group differences were noted in open arm entries.

Analysis of the total activity on the EPM of all groups (see Fig. 1c) revealed no significant differences between the groups $(F(3,118)=0.925, p=0.431)$. Based upon the integrated behavioral measures a significant difference in the anxiety-index was noted between the groups (see Fig. 1d) $(F(3,118)=8.05, p<0.001)$. Animals fed a normal diet and exposed to PSS exhibited significantly greater anxiety ( $p$ value ranging from $<0.001-0.002$ ) than other treatment groups (see Fig. 1d). No other between-group effects were noted.

Acute startle response and startle habituation

Comparisons between the groups in acoustic startle amplitude revealed a significant group difference $(F$ (3, $118)=14.02, p<0.001$ ) (see Fig. 2a). Startle amplitude was significantly greater for animals that were exposed to PSS and fed a normal diet compared to unexposed animals, regardless of diet ( $p$ value $<0.001$ ), as well as for animals exposed to PSS and fed BA compared to unexposed animals fed a normal diet $(p<0.001)$ or supplemented with BA $(p=0.0001)$. In addition, animals that were exposed to PSS and whose diets were supplemented with BA tended to have a lower startle amplitude than exposed animals that were not fed BA $(p=0.085)$. A significant difference was also noted in startle habituation $(F(3,118)=9.11$, $p<0.001$ ) (see Fig. 2b). Animals exposed to PSS regardless of diet had a significantly lower habituation score than all other groups ( $p$ value $<0.001$ ). No other significant differences between groups were noted. 

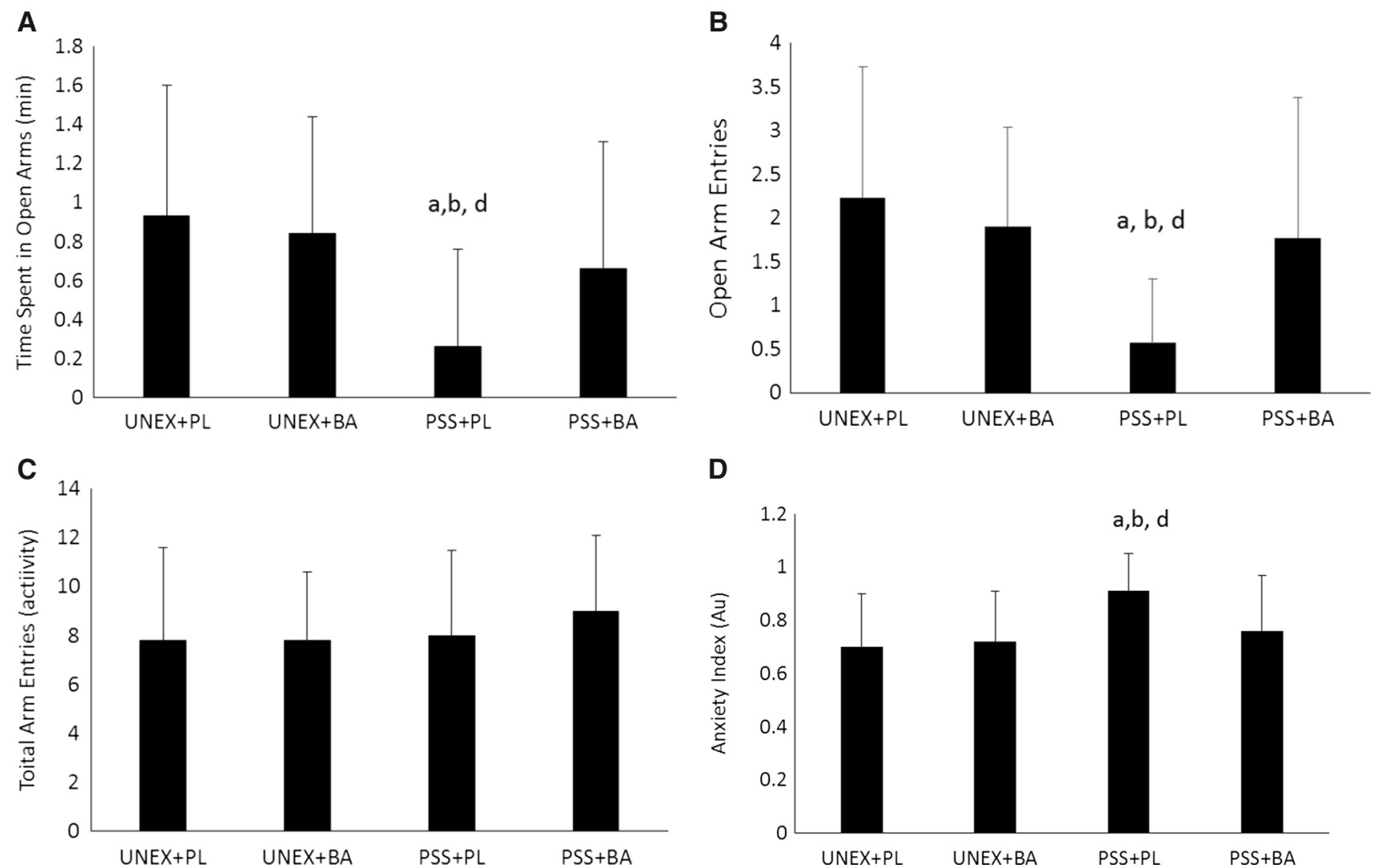

Fig. 1 Behavioral performance in elevated plus-maze. a Comparison between the groups for time spent in open arms; $\mathbf{b}$ comparison between the groups in the number of open arm entries; c comparison between the groups in the total activity; $\mathbf{d}$ comparison between the groups in the anxiety-index; $a$ significantly different than $\mathrm{UNEX}+\mathrm{PL}, b$ significantly different than $\mathrm{UNEX}+\mathrm{BA}, d$ significantly different than PSS $+\mathrm{BA}, U N E X+P L$ animals that were not exposed to

A

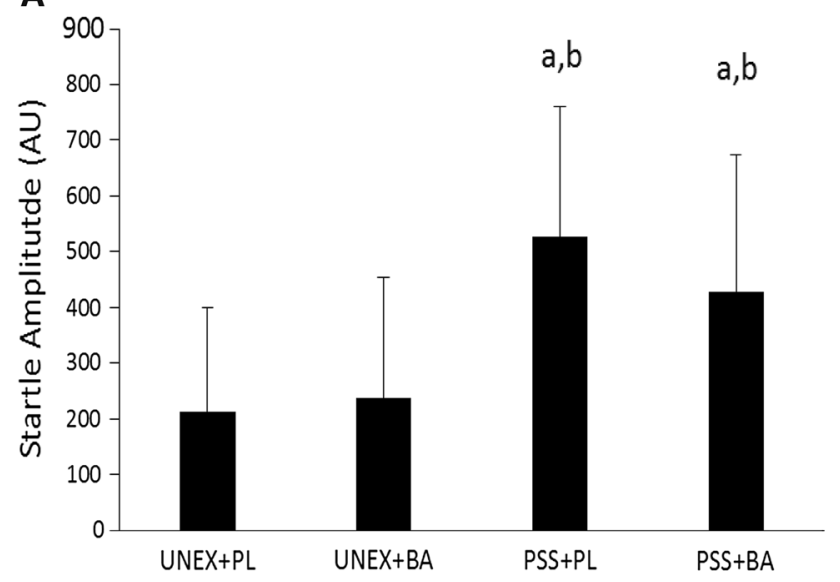

Fig. 2 Acoustic Startle Response. a Comparisons between the groups in startle amplitude; b comparisons between the groups in startle habituation; $a$ significantly different than UNEX+PL, $b$ significantly different than UNEX+BA, $U N E X+P L$ animals that were not exposed to the predator scent stress and fed a normal diet, $U N E X+B A$
B

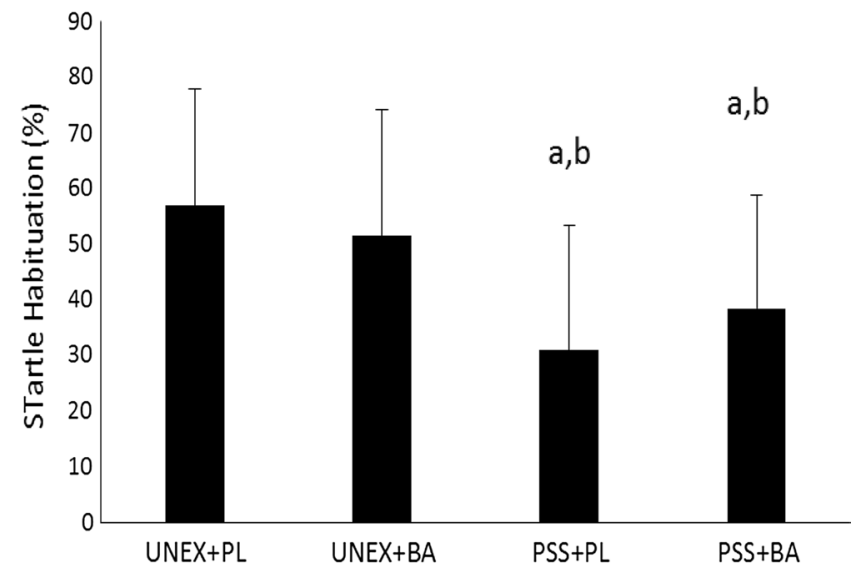

animals that were not exposed to the predator scent stress and supplemented with $\beta$-alanine, $P S S+P L$ animals that were exposed to the predator scent stress and fed a normal diet, $P S S+B A$ animals that were exposed to the predator scent stress and supplemented with $\beta$-alanine. All data reported as mean $\pm \mathrm{SD}$ 


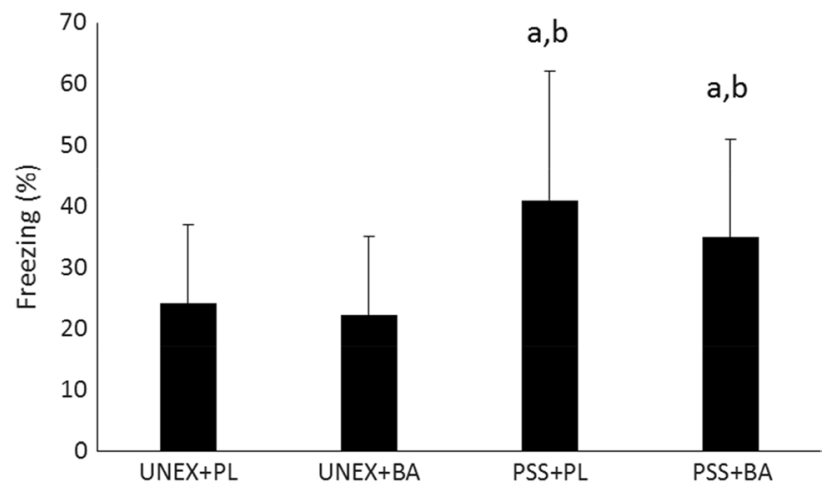

Fig. 3 Freezing Behavior upon Cue-Exposure. a significantly different than UNEX+PL, $b$ significantly different than UNEX+BA, $U N E X+P L$ animals that were not exposed to the predator scent stress and fed a normal diet, $U N E X+B A$ animals that were not exposed to the predator scent stress and supplemented with $\beta$-alanine, $P S S+P L$ animals that were exposed to the predator scent stress and fed a normal diet, $P S S+B A$ animals that were exposed to the predator scent stress and supplemented with $\beta$-alanine. All data reported as mean $\pm \mathrm{SD}$

\section{Effect of cue-exposure on freezing behavior at day 8}

Figure 3 depicts the freezing behavior upon cue-exposure. A significant difference between the groups was seen in freezing behavior $(F(3118)=9.342, p<0.001)$. Animals exposed to PSS and fed a normal diet had significantly greater immobility upon cue than animals that were unexposed and fed a normal diet $(p<0.001)$ or fed a diet supplemented with BA $(p<0.001)$. Animals exposed to PSS and supplemented with BA were also observed to have significantly greater immobility upon cue than animals that were unexposed and fed a normal diet $(p=0.01)$ or fed a diet supplemented with BA $(p=0.003)$. Animals exposed to PSS and fed BA froze approximately $6 \%$ less $(40.9 \%$ versus $34.8 \%$ ) less than animals exposed to PSS and fed a normal diet. However, this difference was not statistically different $(p=0.148)$.

\section{Corticosterone concentrations at day 8 post-PSS exposure}

A significant difference between the groups (Fig. 4) was observed in corticosterone concentrations ( $F$ (3, $76)=13.883, p<0.001)$. Corticosterone concentrations in animals exposed to PSS and fed a normal diet were significantly greater than animals not exposed and fed a similar diet $(p<0.001)$ or supplemented with BA $(p<0.001)$. In addition, animals that were exposed to PSS and supplemented with BA were also observed to have significantly higher corticosterone concentrations than animals that were unexposed and fed a normal diet $(p<0.001)$ or were unexposed and supplemented with BA $(p<0.001)$. No significant differences $(p=0.837)$ were noted between exposed

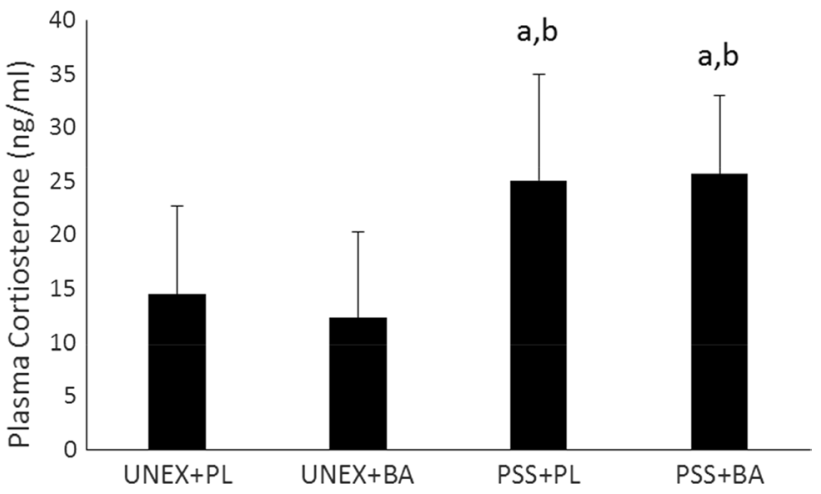

Fig. 4 Corticosterone Concentrations on Day-8 Post-PSS Exposure. $a$ significantly different than UNEX+PL, $b$ significantly different than UNEX+BA, $U N E X+P L$ animals that were not exposed to the predator scent stress and fed a normal diet, $U N E X+B A$ animals that were not exposed to the predator scent stress and supplemented with $\beta$-alanine, $P S S+P L$ animals that were exposed to the predator scent stress and fed a normal diet, $P S S+B A$ animals that were exposed to the predator scent stress and supplemented with $\beta$-alanine. All data reported as mean $\pm \mathrm{SD}$

rats fed a normal diet compared to exposed rats supplemented with BA.

\section{BDNF expression on day-8 post-PSS exposure}

Comparisons between the groups in BDNF expression in the CA1, CA3, and DG subregions can be observed in Fig. 5a-c, respectively. Significant differences were noted in the CA1 $(F(3,36)=23.2, p<0.001)$ and DG subregions $(F(1,36)=4.126, p=0.013)$. No differences were observed between the groups in the CA3 subregion ( $F$ (1, $36)=0.918, p=0.442)$. BDNF expression in the CA1 and DG subregions in the animals that were exposed to PSS and fed a normal diet was significantly lower than that in all other groups ( $p$ values ranging from $<0.001-0.011)$. In addition, BDNF expression in animals exposed to PSS and supplemented with BA was significantly lower than that in unexposed animals ( $r$ values ranging from 0.009-0.024), but significantly greater than that in exposed rats fed a normal diet in the CA1 $(p<0.001)$ only. In the DG subregion, BDNF expression in animals exposed to PSS but supplemented with BA were not statistically different from animals not exposed and either fed a normal diet $(p=0.765)$ or supplemented with BA $(p=0.818)$; however, the expression of BDNF in these animals exposed and fed BA was greater $(p=0.011)$ than that in animals exposed and fed a normal diet. No other differences were noted.

Brain carnosine concentrations on day 8 post-PSS exposure

The concentration of carnosine in various areas of the brain can be observed in Table 1. Significant differences 

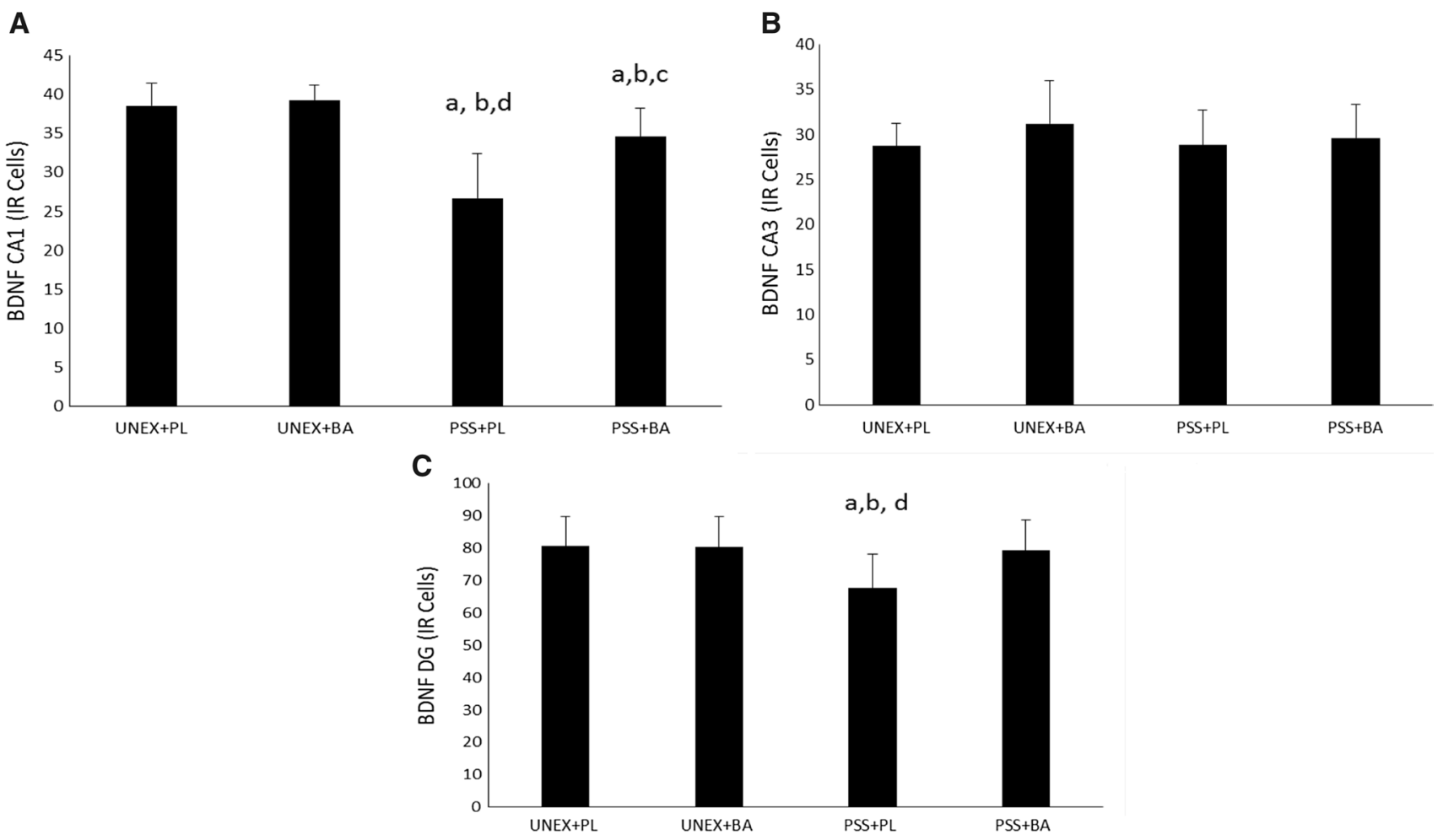

Fig. 5 BDNF Expression on Day-8 Post-PSS Exposure. BDNF expression in the CA1 (a), CA3 (b) and DG (c) subregions. $a$ significantly different than $\mathrm{UNEX}+\mathrm{PL}, b$ significantly different than $\mathrm{UNEX}+\mathrm{BA}, c$ significantly different than PSS $+\mathrm{PL}, d$ significantly different than PSS+BA, $U N E X+P L$ animals that were not exposed to the predator scent stress and fed a normal diet, $U N E X+B A$ animals that were not exposed to the predator scent stress and supplemented with $\beta$-alanine, $P S S+P L$ animals that were exposed to the predator scent stress and fed a normal diet, $P S S+B A$ animals that were exposed to the predator scent stress and supplemented with $\beta$-alanine. All data reported as mean $\pm \mathrm{SD}$

Table 1 Brain carnosine concentrations $\left(\mathrm{mg} \mathrm{L}^{-1}\right)$

\begin{tabular}{lllllrr}
\hline & Hippocampus & Cortex & Hypothalamus & Amygdala & Olfactory bulb & Thalamus \\
\hline UNEX+PL & $0.71 \pm 0.59$ & $0.76 \pm 0.79$ & $0.64 \pm 0.64$ & $0.30 \pm 0.34$ & $11,011 \pm 3910$ & $0.77 \pm 0.46$ \\
UNEX+BA & $3.75 \pm 3.98^{\mathrm{a}}$ & $3.78 \pm 4.47^{\mathrm{a}}$ & $2.60 \pm 2.85$ & $2.34 \pm 3.04$ & $8309 \pm 6887$ & $3.99 \pm 5.10^{\mathrm{a}}$ \\
PSS+PL & $1.12 \pm 0.90$ & $0.96 \pm 0.43$ & $0.78 \pm 0.46$ & $0.55 \pm 0.50$ & $5744 \pm 6491$ & $1.16 \pm 0.70$ \\
PSS+BA & $5.58 \pm 4.39^{\mathrm{a}, \mathrm{b}}$ & $6.55 \pm 4.90^{\mathrm{a}, \mathrm{b}}$ & $4.54 \pm 4.04^{\mathrm{a}, \mathrm{b}}$ & $3.52 \pm 3.84^{\mathrm{a}, \mathrm{b}}$ & $13,109 \pm 9773$ & $5.97 \pm 4.48^{\mathrm{a}, \mathrm{b}}$ \\
\hline
\end{tabular}

All data reported as mean $\pm \mathrm{SD} U N E X+P L$ animals that were not exposed to the predator scent stress and fed a normal diet, $U N E X+B A$ animals that were not exposed to the predator scent stress and supplemented with $\beta$-alanine, $P S S+P L$ animals that were exposed to the predator scent stress and fed a normal diet, $P S S+B A$ animals that were exposed to the predator scent stress and supplemented with $\beta$-alanine

a significantly different than UNEX+PL

b significantly different than PSS + PL

were noted in the hippocampus $(F(3,36)=5.818$, $p=0.002)$, cerebral cortex $(F(1,36)=6.659, p=0.001$, hypothalamus $(F(3,36)=5.348, p=0.004)$, amygdala $(F(3,36)=3.825, p=0.018)$, and thalamus $(F$ $(3,36)=5.162, p=0.005)$. No significant differences were noted in the olfactory bulb $(F(3,36)=2.051$, $p=0.124)$. In the hippocampus, cortex, hypothalamus, amygdala, and thalamus of animals that ingested BA and were exposed to PSS had significantly greater carnosine concentrations ( $p$ value ranging from $<0.001-0.011$ ) than animals who were fed a normal diet, regardless of exposure. Animals that were not exposed to PSS and supplemented with BA had a significantly greater carnosine concentration in the hippocampus $(p=0.030)$ and thalamus $(p=0.042)$ compared to animals not exposed and fed a normal diet. Additional comparisons between animals that were not exposed to PSS and supplemented with BA appeared to result in only strong trends ( $p$ value ranging from 0.051 to 0.089 ) when compared to animals fed a normal diet, regardless of exposure, in the cerebral 
cortex, hypothalamus, and amygdala. No differences were noted in comparisons between animals unexposed and fed BA compared to animals exposed and fed a normal diet in the hypothalamus $(p=0.113)$ and amygdala $(p=0.115)$ only.

\section{Discussion}

The results of this study indicated that 30 days of $\beta$-alanine ingestion in rats was effective in attenuating some of the behaviors tested and associated with exposure to PSS. Rats fed a normal diet and exposed to PSS were observed to be significantly less active when placed in the elevated maze and had a greater anxiety level compared to animals that were either unexposed, or animals that were exposed and supplemented with BA. However, $\beta$-alanine supplementation to the diet was unable to alleviate all of the behaviors associated with exposure to stress. Animals exposed to PSS were shown to experience a significantly elevated startle response, freezing and a lower startle habituation than animals that were not exposed, regardless of whether they were supplemented with $\beta$-alanine or not. Nonetheless, animals that were exposed but supplemented with $\beta$-alanine demonstrated a $19 \%$ lower startle response $(p=0.085)$ and a $15 \%$ lower freezing response $(p=0.148)$ than animals exposed and which were not supplemented.

The animal model of PTSD used in this study has been previously established as a valid and effective method of examining biomolecular and physiological parameters of specific response patterns of stress (Cohen et al. 2012a; Matar et al. 2013). Although this model has not been previously used for $\beta$-alanine ingestion, it has been established as an effective approach to determine the effects of various interventions on the behavioral response to stress (Cohen et al. 2012a). In comparison to other studies, using this same stress model in rats, differences in the startle amplitude response (2.5 to 2.2 fold higher) seen between $\mathrm{PSS}+\mathrm{PL}$ versus $\mathrm{UNEX}+\mathrm{PL}$ or $\mathrm{UNEX}+\mathrm{BA}$, respectively, was consistent with the 2.5 -fold increase previously reported by Cohen et al. (2004). Exposure to PSS in this study also resulted in a $30 \%$ elevation in anxiety index and a nearly twofold increase in the freezing response compared to animals unexposed. These differences were similar to that reported by Cohen et al. (2012b), but lower in magnitude than that reported in the previous work by the same group (Cohen et al. 2004). The results of this study were also similar to other rodent models using a different PTSD model (Solanki et al. 2015). Those investigators using a single-prolonged stress model that incorporated psychological, physical, and pharmaceutical stresses also showed significant reductions in the activity patterns of rats in the
EPM and a greater time of immobility or freezing when placed in a water tank.

Exposure to PSS was also accompanied by significant elevations in plasma corticosterone concentrations 7-days following the stress. This was consistent with other studies using the same stress model (Cohen et al. 2012b; Kozlovsky et al. 2007). Elevations in glucocorticoids are associated with exposure to stress (Myers et al. 2014), but recent evidence suggests that elevations in glucocorticoids may play a role in enhancing versus impairing recovery (Zohar et al. 2011). Previous research has indicated that a blunted hypothalamic-pituitary adrenal axis response to an acute stress may increase the susceptibility for PTSDlike symptoms (Cohen et al. 2006; McFarlane et al. 2011), which can be reversed with treatment of high-dose cortisone administration (Cohen et al. 2006; Kim et al. 2014; Zohar et al. 2011). However, glucocorticoid concentrations appear to demonstrate an inverted U effect. Although low concentrations may be a predictor for PTSD, high concentrations have also been suggested to be associated with morphological changes of neurons that also contribute to a negative behavioral response seen during stress (Sebastian et al. 2013). The hippocampus is sensitive to both acute and chronic stress. A chronic stress response may elevate glucocorticoid expression in the brain causing the neurons in the hippocampus to undergo reversible remodeling, especially in the DG-CA3 regions (McEwen 2007). These changes often involve dendritic atrophy (Romeo et al. 2004), which may result in diminished function in hippocampal-dependent memory tasks (Coburn-Litvak et al. 2003). In contrast, BDNF is reported to have the opposite effect on neuroplasticity by enhancing neurogenesis and dendritic remodeling (Yao et al. 2011). Elevations in corticosterone concentrations observed in both groups of rats exposed to PSS in this study were likely indicative of the stress experienced by those animals. However, the animals that were supplemented with BA appeared to recover or demonstrated a greater resiliency to stress in regard to their levels of anxiety and performance in the EPM. This may have been a function of both elevations in brain carnosine levels and an increased expression of BDNF. This is supported by the association seen in the increased expression of BDNF in the CA1 and DG subregions observed in this study with a reduction in the startle response $(r=-0.341$, $p=0.031$ and $r=-0.523, p=0.001$, respectively) and freezing response $(r=-0.572, p<0.001$ and $r=-0.335$, $p=0.035$, respectively) of the animals examined.

Rats supplemented with $\beta$-alanine in this study experienced significant elevations in carnosine concentrations in nearly all segments of the brain. Supplementation with $\beta$-alanine did not appear to have any influence on carnosine concentrations in the olfactory bulb. This was likely related to the high concentrations of carnosine generally 
seen in the olfactory bulb of most mammalian species (Bonfanti et al. 1999). However, differences in carnosine concentrations within the other brain regions support previous studies that showed $\beta$-alanine supplemented diets can increase brain carnosine concentrations in mice (Murakami and Furuse 2010) and chickens (Tomonaga et al. 2005, 2012). Tomonaga et al. (2008) suggested that brain carnosine can attenuate depression-like behavior by reducing brain metabolites of norepinephrine, or through stimulation of histaminergic neurons through one of its constituents; histidine. Others have suggested that carnosine might influence brain antioxidant activity (Kohen et al. 1988), while others have reported that elevations in brain carnosine concentrations in the hypothalamus and cerebral cortex are associated with increases in the concentration of BDNF in the hippocampus of mice (Murakami and Furuse 2010). In the present study, elevations in brain carnosine concentrations in the PSS+BA group were associated with maintaining expression of BDNF in the CA1 and DG subregions of the hippocampus compared to PSS+PL rats. Elevations in carnosine concentrations in the hippocampus, cortex, hypothalamus, amygdala ++ and thalamus were inversely associated with anxiety index ( $r$ value ranging from -0.471 to $-0.550, p$ value $<0.002$ ) and positively associated with improved time spent in the open arms ( $r$ value ranging from 0.453 to $0.521, p$ value $<0.003$ ).

The significant decrease in BDNF expression observed in rodents exposed to PSS and fed a normal diet is consistent with previous studies reporting an attenuation of BDNF expression and PTSD-like behavioral stress response (Kozlovsky et al. 2007; Zohar et al. 2011). Although $\beta$-alanine ingestion and subsequent carnosine synthesis in the brain did not appear to increase BDNF expression in unexposed rats, it did appear to maintain BDNF expression in those rats that were exposed to PSS. Similar expression of BDNF expression in both UNEX+BA and UNEX+PL suggested that elevations in brain carnosine levels were not directly responsible for altering BDNF expression. These results on the surface appear to contrast with those of Murakami and Furuse (2010) who showed significant elevations in BDNF concentrations in hippocampus homogenates following $\beta$-alanine ingestion in physically stressed mice. However, those investigators did not use a nonstressed group as a control. In the present study, when comparing both groups of animals that were exposed to PSS, and differing only in regard to whether supplemented with $\beta$-alanine, the results confirm the findings of Murakami and Furuse (2010). The mechanism of elevated brain carnosine and maintenance of BDNF expression in the hippocampus is not well understood, but it may be related to its role as a neural protectant through its action as an antioxidant (Kohen et al. 1988). Oxidative stress and inflammation in the brain have been demonstrated to cause the development and further exacerbation of PTSD (Wilson et al. 2013). Thus, carnosine's role as an antioxidant may prevent the neurodegeneration associated with elevated glucocorticoids and indirectly presere BDNF expression. Further research is warranted to examine the role of carnosine and oxidative stress in brain tissue.

In summary, the results of this study indicate that 30-day of $\beta$-alanine supplementation appears to promote resiliency and/or recovery from PSS. The protective effects associated with elevations in brain carnosine appear to be related to a protection of BDNF expression in the hippocampus. The precise mechanism of how elevated carnosine concentrations support BDNF expression requires additional research. This appears to be the first study known to demonstrate a potential role of $\beta$-alanine as a dietary supplement for the treatment or prevention of PTSD.

Acknowledgments The authors would like to thank Natural Alternatives International (San Marcos, CA, USA) for providing support for this study.

Conflict of interest Roger Harris is a paid consultant to NAI and is named on a number of patents regarding $\beta$-alanine use. All other authors declare no conflict of interest.

Open Access This article is distributed under the terms of the Creative Commons Attribution License which permits any use, distribution, and reproduction in any medium, provided the original author(s) and the source are credited.

\section{References}

Aldini G, Orioli M, Carini M, Facino RM (2004) Profiling histidinecontaining dipeptides in rat tissues by liquid chromatography/ electrospray ionization tandem mass spectrometry. J Mass Spectrom 39:1417-1428

American Psychiatric Association (1994) Diagnostic and statistical manual of mental disorders, 4th edn. American Psychiatric Association, Washington, DC

American Psychiatric Association (2013) Diagnostic and statistical manual of mental disorders, 5th edn. American Psychiatric Association, Washington, DC

Angelucci F, Ricci V, Gelfo F, Martinotti G, Brunetti M, Sepede G, Signorelli M, Aguglia E, Pettorruso M, Vellante F, Di Giannantonio M, Caltagirone C (2014) BDNF serum levels in subjects developing or not post-traumatic stress disorder after trauma exposure. Brain Cogn 84:118-122

Bisson JI, Shepherd JP, Joy D, Probert R, Newcombe RG (2004) Early cognitive-behavioural therapy for post-traumatic stress symptoms after physical injury. Randomized controlled trial. $\mathrm{Br}$ J Psychiatry 184:63-69

Bonfanti L, Peretto P, De Marchis S, Fasolo A (1999) Carnosinerelated dipeptides in the mammalian brain. Prog Neurobiol 59:333-353

Bryant RA, Mastrodomenico J, Felmingham KL, Hopwood S, Kenny L, Kandris E, Cahill C, Creamer M (2008) Treatment of acute stress disorder: a randomized controlled trial. Arch Gen Psychiatry 65:659-703 
Bryant RA, Creamer M, O’Donnell M, Silove D, McFarlane AC, Forbes D (2014) A comparison of the capacity of DSM-IV and DSM-5 acute stress disorder definitions to predict posttraumatic stress disorder and related disorders. J Clin Psychiatry. doi: 10.4088/JCP.13m08731

Castrén E, Rantamäki T (2010) The role of BDNF and its receptors in depression and antidepressant drug action: reactivation of developmental plasticity. Dev Neurobiol 70:289-297

Coburn-Litvak PS, Pothakos K, Tata DA, McCloskey DP, Anderson BJ (2003) Chronic administration of corticosterone impairs spatial reference memory before spatial working memory in rats. Neurobiol Learn Mem 80:11-23

Cohen H, Zohar J (2004) An animal model of posttraumatic stress disorder: the use of cut-off behavioral criteria. Ann NY Acad Sci 1032:167-178

Cohen H, Zohar J, Matar M (2003) The relevance of differential response to trauma in an animal model of posttraumatic stress disorder. Biol Psychiatry 53:463-473

Cohen H, Zohar J, Matar MA, Zeev K, Loewenthal U, Richter-Levin G (2004) Setting apart the affected: the use of behavioral criteria in animal models of post-traumatic stress disorder. Neuropsychopharmacology 29:1962-1970

Cohen H, Zohar J, Gidron Y, Matar MA, Belkind D, Loewenthal U, Kozlovsky N, Kaplan Z (2006) Blunted HPA axis response to stress influences susceptibility to posttraumatic stress response in rats. Biol Psychiatry 59:1208-1218

Cohen H, Kozlovsky N, Cramer A, Matar MA, Zohar J (2012a) Animal model for PTSD: from clinical concept to translational research. Neuropharmacology 62:715-724

Cohen H, Liu T, Kozlovsky N, Kaplan Z, Zohar J, Mathé AA (2012b) The neuropeptide Y (NPY)-ergic system is associated with behavioral resilience to stress exposure in an animal model of post-traumatic stress disorder. Neuropsychopharmacology. 37:350-363

Fuxe K, Cintra A, Agnati LF, Harfstrand A, Wikstrom AC, Okret S, Zoli M, Miller LS, Greene JL, Gustafsson JA (1987) Studies on the cellular localization and distribution of glucocorticoid receptor and estrogen receptor immunoreactivity in the central nervous system of the rat and their relationship to the monoaminergic and peptidergic neurons of the brain. J Steroid Biochem 27:159-170

Kaouane N, Porte Y, VAllee M, Brayda-Bruno L, Mons N, Calandreau L, Marighetto A, Piazza PV, Desmedt A (2012) Glucocorticoids can induce PTSD-like memory impairements in mice. Science 335:1510-1513

Kim JJ, Fanselow MS, DeCola JP, Landeira-Fernandez J (1992) Selective impairment of long-term but not short-term conditional fear by the N-methyl-D-aspartate antagonist APV. Behav Neurosci 106:591-596

Kim H, Yi JH, Choi K, Hong S, Shin KS, Kang SJ (2014) Regional differences in acute corticosterone-induced dendritic remodeling in the rat brain and their behavioral consequences. BMC Neurosci 15:65

Kirkpatrick HA, Heller GM (2014) Post-traumatic stress disorder: theory and treatment update. Int J Psychiatry Med 47:337-346

Kohen R, Yamamoto Y, Cundy KC, Ames BN (1988) Antioxidant activity of carnosine, homocarnosine, and anserine present in muscle and brain. Proc Natl Acad Sci 85:3175-3179

Kozlovsky N, Matar MA, Kaplan Z, Kotler M, Zohar J, Cohen H (2007) Long-term down-regulation of BDNF mRNA in rat hippocampal CA1 subregion correlates with PTSD-like behavioral stress response. Int J Neuropsychopharmacol 10:741-758

Matar MA, Zohar J, Cohen H (2013) Translationally relevant modeling of PTSD in rodents. Cell Tissue Res 354:127-139

McEwen BS (1999) Stress and hippocampal plasticity. Annu Rev Neurosci 22:105-122

McEwen BS (2007) Physiology and neurobiology of stress and adaptation: central role of the brain. Physiol Rev 87:873-904
McFarlane AC, Barton CA, Yehuda R, Wittert G (2011) Cortisol response to acute trauma and risk of posttraumatic stress disorder. Psychoneuroendocrinology 36:720-727

Milad M, Quirk GJ (2002) Neurons in medial prefrontal cortex signal memory for fear extinction. Nature 420:70-74

Miller MM, McEwen BS (2006) Establishing an agenda for translational research on PTSD. Ann NY Acad Sci 1071:294-312

Murakami T, Furuse M (2010) The impact of taurine- and betaalanine-supplemented diets on behavioral and neurochemical parameters in mice: antidepressant versus anxiolytic-like effects. Amino Acids 39:427-434

Myers B, McKlveen JM, Herman JP (2014) Glucocorticoid actions on synapses, circuits, and behavior: implications for the energetics of stress. Front Neuroendocrinol 35:180-196

Richardson MP, Strange BA, Dolan RJ (2004) Encoding of emotional memories depends on amygdala and hippocampus and their interactions. Nat Neurosci 7:278-285

Romeo RD, Waters EM, McEwen BS (2004) Steroid-induced hippocampal synaptic plasticity: sex differences and similarities. Neuron Glia Biol 1:219-229

Sebastian V, Estil JB, Chen D, Schrott LM, Serrano PA (2013) Acute physiological stress promotes clustering of synaptic markers and alters spine morphology in the hippocampus. PLoS One 8:e79077

Solanki N, Alkadhi I, Atrooz F, Patki G, Salim S (2015) Grape powder prevents cognitive, behavioral, and biochemical impairments in a rat model of posttraumatic stress disorder. Nutr Res 35:65-75

Sousa N, Madeira MD, Paula-Barbosa MM (1998) Effects of corticosterone treatment and rehabilitation on the hippocampal formation of neonatal and adult rats. An unbiased stereological study. Brain Res 794:199-210

Stewart MG, Davies HA, Sandi C, Kraev IV, Rogachevsky VV, Peddie CJ, Rodriguez JJ, Cordero MI, Donohue HS, Gabbott PL, Popov VI (2005) Stress suppresses and learning induces plasticity in CA3 of rat hippocampus: a three dimensional ultrastructural study of thorny excrescences and their postsynaptic densities. Neuroscience 131:43-54

Takeuchi K, Toyohama H, Sakaquchi M (2000) A hyperosmotic stress-induced mRNA of carp cell encodes $\mathrm{NA}(+)$ - and $\mathrm{Cl}(-)$ dependent high affinity taurine transporter. Biochim Biophys Acta 1464:219-230

Tomonaga S, Tachibana T, Takagi T, Saito ES, Zhang R, Denbow DM, Furuse M (2004) Effect of central administration of carnosine and its constituents on behaviors in chicks. Brain Res Bull 63:75-82

Tomonaga S, Kaji Y, Tachibana T, Denbow DM, Furuse M (2005) Oral administration of $\beta$-alanine modifies carnosine concentrations in the muscles and brains of chicken. Anim Sci J 76:249-254

Tomonaga S, Yamane H, Onitsuka E, Yamada S, Sato M, Takahata Y, Morimatsu F, Furuse M (2008) Carnosine-induced anti-depressant-like activity in rats. Pharmacol Biochem Behav 89:627-632

Tomonaga S, Matsumoto M, Furuse M (2012) $\beta$-Alanine enhances brain and muscle carnosine levels in broiler chicks. J Poult Sci 49:308-312

Wilson CB, McLaughlin LD, Nair A, Ebenezer PJ, Dange R, Francis $\mathbf{J}$ (2013) Inflammation and oxidative stress are elevated in the brain, blood, and adrenal glands during the progression of posttraumatic stress disorder in a predator exposure animal model. PLoS One 8:e76146

Yao SY, Lau BW, Tong JB, Wong R, Ching YP, Qui G, Tang SW, Lee TMC, So KF (2011) Hippocampal neurogenesis and dendritic plasticity support running-improved spatial learning and depression-like behavior in stressed rats. PLoS One 6:e24263

Zohar J, Yahalom H, Kozlovsky N, Cwikel-Hamzany S, Matar MA, Kaplan Z, Yehuda R, Cohen H (2011) High dose hydrocortisone immediately after trauma may alter the trajectory of PTSD: interplay between clinical and animal studies. Eur Neuropsychopharmacol 21:796-809 